\title{
Optimization Analysis of Initial Sheet Metal Contour Line for High-Strength Boron Steel in Hot Stamping
}

\author{
Z. Q. Zhang, ${ }^{1}$ X. F. Jia, Y. J. Wang, and P. Gao \\ College of Materials Science and Engineering, Jilin University, Changchun, China \\ ${ }^{1}$ Zhangzq@jlu.edu.cn
}

Based on the principle of hot stamping, a simulation model of hot stamping was established. The blank contour line of $U$ part was optimized by using a multiple iterative algorithm. By comparison with the simulation and experimental data, the multiple iterative optimization algorithm was verified. Moreover, the blank contour lines of a bumper and the B-pillar of Numisheet 2008 were optimized. The result showed that the multiple iterative method for optimizing initial blank contour line had high calculating speed and precision. The hot stamping parts' contour lines match the ideal part contour lines quite well.

Keywords: high strength boron steel, hot stamping, optimization analysis, contour line, finite element method.

Introduction. Hot stamping technology is the key to the lightweight of automobile and has been increasingly used in manufacturing inside door beam, roof rail, bumper, $\mathrm{A} / \mathrm{B} / \mathrm{C}$-pillar reinforcements, etc. $[1,2]$. Accurate design of initial blank contour line can reduce the possibility of forming defects and improve the part quality $[3,4]$. Scientists have got many computing methods under different assumptions such as velocity field method [5], slip line field method [6, 7], geometric mapping [8], potential field simulation [9, 10] for cold stamping process. However, hot stamping process is a thermal-mechanical coupling process. One step finite element inverse algorithm is mainly used in hot stamping to get the initial blank contour line and redundant materials need to be removed by laser cutting after hot stamping. It not only wastes raw materials but also results in low production efficiency. Based on multiple iterative inverse algorithms, the process of hot stamping was simulated and the optimized blank initial contour line was obtained by establishing an optimized model, which required no further application of a cutting process after hot stamping.

1. Process of Blank Initial Contour Optimization. In the process of optimization, the border line of the part is the target object, where the border line of simulated part is regarded as comparative object, while the blank initial contour line is the optimization object. An optimal contour line is chosen from many calculating contour lines through simulation. In simulation, one-step finite element inverse algorithm is employed to get initial blank contour line and then parameters' editor is initialized to mesh the whole model and set parameters, input cycle definition and related control parameters, such as number of cycle, object choice, coefficient of thermal expansion, etc. All those procedures can help to establish an optimized model and offer the reference for the following iterative computation. If an optimization meets the demands of presupposed forming precision, iteration will stop. If not, further optimization will go on after establishing the new mesh within the maximum iterations. The control process of the optimization model is shown in Fig. 1.

\section{Contour Optimization and Forming Experiment of U-Shaped Part.}

2.1. Contour Optimization of $\boldsymbol{U}$-Shaped Part. The ideal U-shaped part has a length of $54 \mathrm{~mm}$, width of $10 \mathrm{~mm}$ and height of $15 \mathrm{~mm}$. One-step inverse algorithm is used to get the blank initial contour line and then generate the blank with the thickness of $1 \mathrm{~mm}$. The position of the blank and die before forming is shown in Fig. 2. The initial blank mesh was 


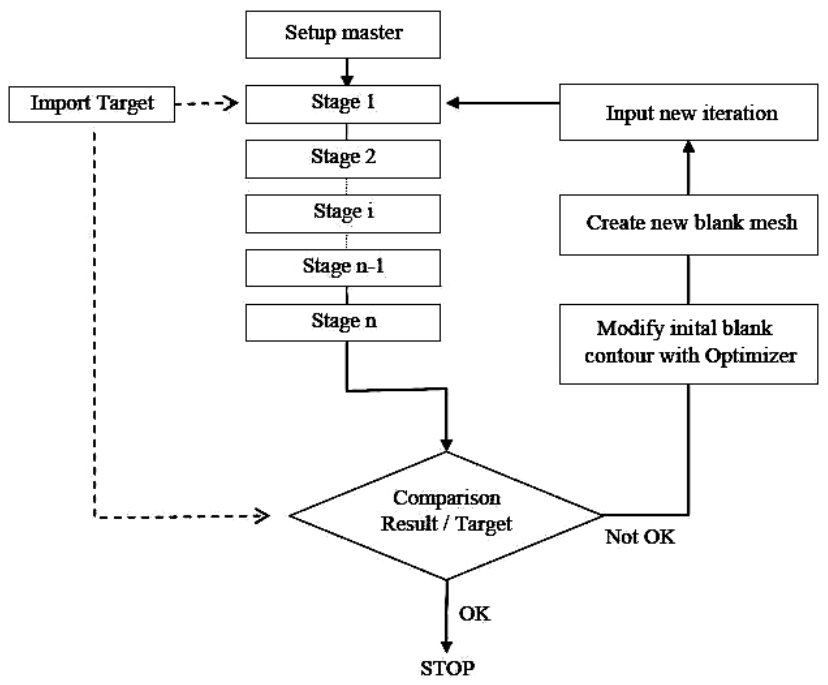

Fig. 1. Schematic diagram of the control process for optimization model.

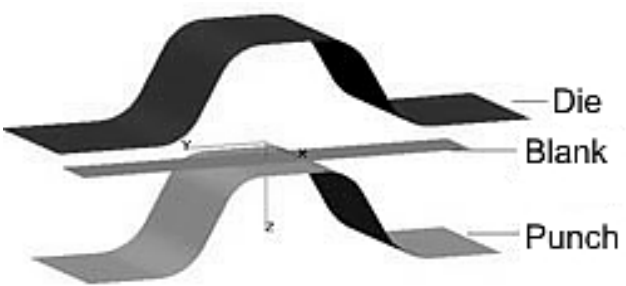

Fig. 2. Hot stamping of U-shape part.

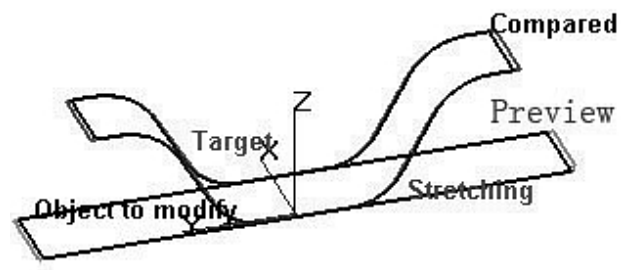

Fig. 3. Contour lines of ideal part, formed part and initial blank.

generated with 3131 nodes and 2592 elements by finite element meshing. The material model should be a temperature and strain-rate dependent elastic-plastic model considering phase transformation and thermal expand. Stress-strain curves of the material at various temperatures are from Numisheet 2008 [11]. Figure 3 shows the contour line position of the ideal part (target), formed part (compared), and the initial blank (object to modify). Preview is the initial blank contour line obtained from multiple iterative inverse algorithms.

Figure 4 shows the size deviation distribution of the formed part before and after optimization. Before optimization, contour line of formed part strongly deviated from that of the ideal part. Within the tolerance $0.5 \mathrm{~mm}$ range, the contour line agreement of the formed part and the ideal part was $88.22 \%$ and dimensional deviation at flange boundary was the biggest, and the maximum was $0.84 \mathrm{~mm}$. After the optimization, the contour line agreement of the formed part and the ideal part reached 100\%. Dimensional accuracy of the part was greatly improved. 

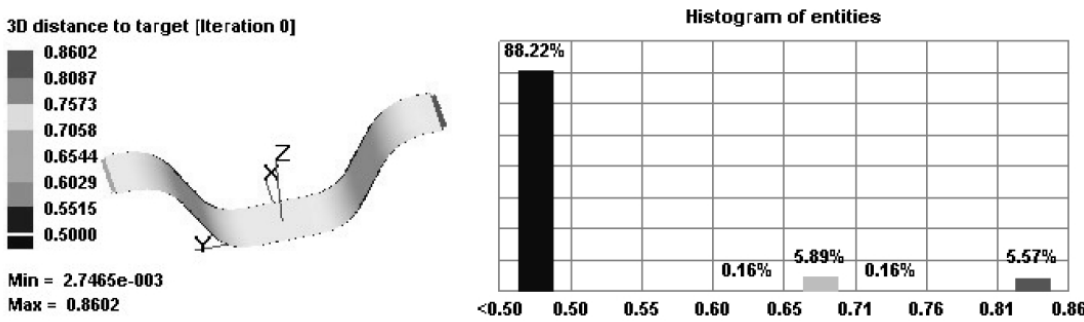

a
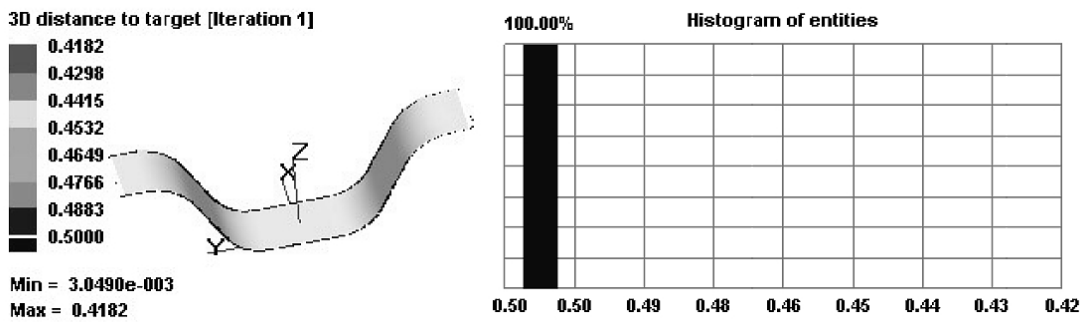

$\operatorname{Min}=3.0490 \mathrm{e}-003$

Max $=\mathbf{0 . 4 1 8 2}$

b

Fig. 4. Contour line size deviation distribution histogram of formed part and ideal part before (a) and after optimization (b).

2.2. Experiment on Forming U-Shaped Part. The material used in this study is $\mathrm{B} 1500 \mathrm{HS}$, which is manufactured by Bao steel Group. Table 1 shows the composition of this material.

$\mathrm{T}$ a b 1 e 1

\section{Chemical Composition of B1500HS Steel (wt.\%)}

\begin{tabular}{|c|c|c|c|c|c|}
\hline $\mathrm{C}$ & $\mathrm{Mn}$ & $\mathrm{Cr}$ & $\mathrm{Si}$ & $\mathrm{Ti}$ & $\mathrm{B}$ \\
\hline 0.22 & 1.20 & 0.20 & 0.20 & 0.30 & 0.05 \\
\hline
\end{tabular}

The experiment uses DNS100 electronic universal testing machine and the heating device is SX2-5-12 resistance furnace. B1500HS with a thickness of $1 \mathrm{~mm}$ was cut along the optimized contour line and placed into the furnace that had been heated to $930^{\circ} \mathrm{C}$ to keep for $3 \mathrm{~min}$. The heated blank was removed quickly from the furnace to the fixed die, and then the punch descended very quickly to form the blank. The temperature of tools is $20^{\circ} \mathrm{C}$ and the stamping force is $6 \mathrm{kN}$.

The dimensions of the formed part, the optimized part and the ideal part are shown in Table 2. Compared with the ideal part, the optimized part had a small contour line size deviation that was within $0.5 \mathrm{~mm}$ and it is accepted by dimensional tolerance. Small flange and low pressure occurred in the process might cause a little spring back. Figure 5 shows the U-shaped part.

$\mathrm{T}$ a b 1 e 2

Comparison of the Optimized Contour and the Ideal Contour

\begin{tabular}{||c|c|c|c|c||}
\hline Characteristic & Tested part & Simulated part & Ideal part & Error \\
\hline Length $(\mathrm{mm})$ & 53.0 & 53.29 & 53.33 & -0.33 \\
\hline Height $(\mathrm{mm} 0$ & 15.4 & 15.00 & 15.00 & 0.40 \\
\hline
\end{tabular}




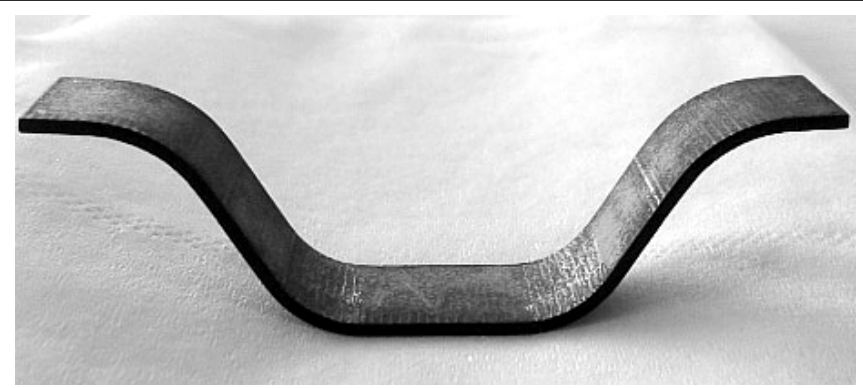

Fig. 5. U-shaped part after stamping.

\section{Test Verification.}

3.1. Hot Stamping of Bumper and Optimization of the Blank Initial Contour Line. Hot stamping process of a bumper was simulated and the blank used in the study has a thickness of $1.5 \mathrm{~mm}$ and a friction coefficient of 0.35 . The blank mesh had 13,441 nodes and 35,095 elements. The blank initial contour line was obtained by finite element inverse algorithm. Formed part did not meet the demands of dimensional precision and the deviation mainly focused on the both ends of the long axis, which is shown in Fig. 6a. The dimensional tolerance of the bumper is $0.5 \mathrm{~mm}$. At the initial step of optimization, the contour lines of formed part had a lower agreement that was $0.85 \%$. The dimensional deviation was largely from $0.5 \mathrm{~mm}$ to the maximum $16.31 \mathrm{~mm}$, which did not meet the demands of the dimensional precision. After four times optimized iterative computations, the contour lines of formed part had an agreement that was $95.01 \%$. The maximum dimensional deviation was under $0.54 \mathrm{~mm}$, which is shown in Fig. 6b. When the presupposed dimensional precision was reached, iterative computation would stop, and the optimized initial contour line of the bumper could be obtained.

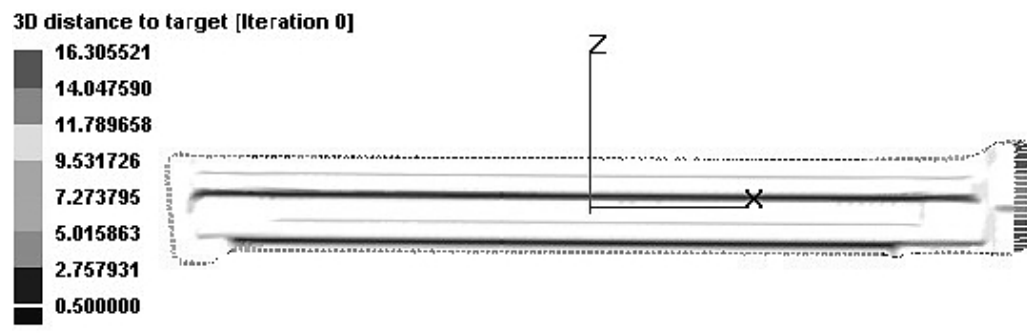

Min $=0.044290$

$\operatorname{Max}=16.305521$

a

3D distance to target [Iteration 4]

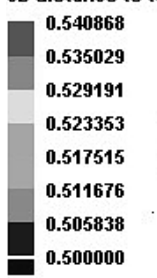

Min $=\mathbf{0 . 0 1 0 2 0 6}$

Max $=0.540868$

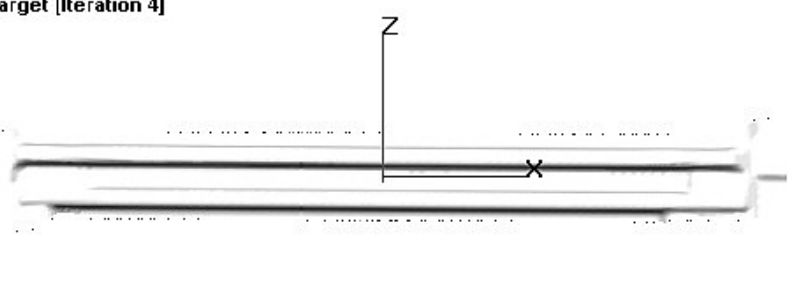

$\mathrm{b}$

Fig. 6. Contour line size deviation distribution of formed and ideal part before (a) and after (b) optimization. 


\subsection{Hot Stamping of B-Pillar and Partial Optimization of the Blank Initial Contour}

Line. Finite element simulation model of a B-pillar was established, and the material used in the study is $22 \mathrm{MnB} 5$, which has a thickness of $1.95 \mathrm{~mm}$. Because the material flow of B-pillar in the forming process is rather complicated, optimization of the whole blank initial contour line will not get an ideal result and thus partial optimization of the blank initial contour line can be carried out and laser edge cutting can also be reduced greatly. The B-pillar in the study didn't match the size of the ideal part due to deep drawing in the forming process. Three points $\mathrm{A}, \mathrm{B}$, and $\mathrm{C}$ were adopted along the contour line of the part and this part of contour line was ignored (red color), shown in Fig. 7. Hot stamping forming model of B-pillar was established, and the beam initial contour line was optimized. Iterations were 8 and dimension tolerance was $0.7 \mathrm{~mm}$.

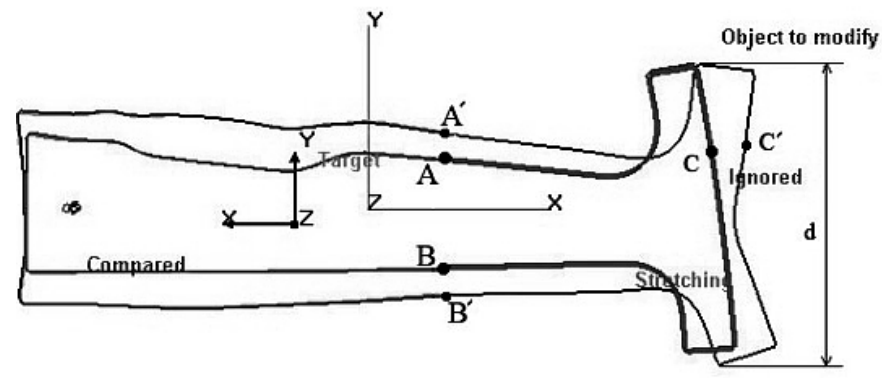

Fig. 7. Schematic diagram of B pillar partial optimization.

Before the optimization, the contour line agreement of formed part and ideal part was $34.68 \%$, and their maximum deviation was $2.79 \mathrm{~mm}$. After six iterations, the agreement reached $93.83 \%$, and their maximum deviation was $1.14 \mathrm{~mm}$ (Fig. 8). The dimensional accuracy of the formed part was strongly improved.
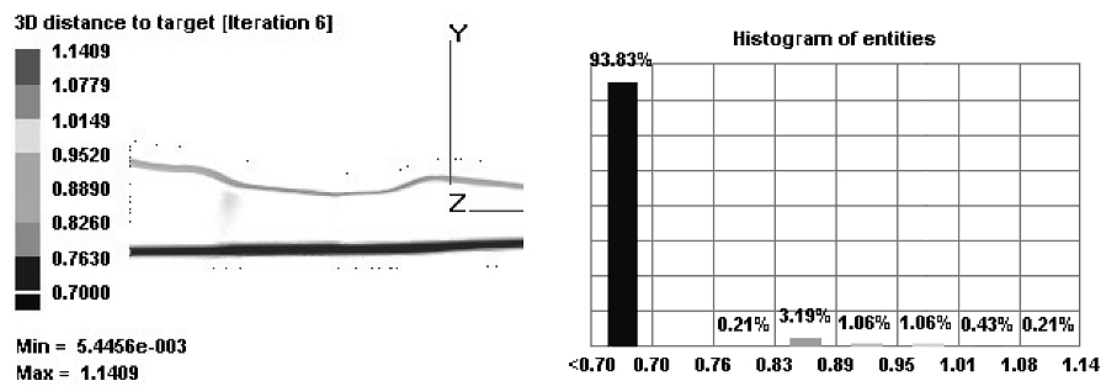

Fig. 8. Contour line size deviation distribution histogram of formed part and ideal part.

Conclusions. Based on the hot stamping principle, blank initial contour line of a U-shape part was optimized by multiple iterative inverse algorithms. The results show that the contour line agreement of formed part and ideal part has been greatly improved and the hot stamping experimental data of U-shape part verify the effectiveness of this method. The total optimization of bumper contour line and partial optimization of B-pillar contour line also provided good results. Partial optimization covers the shortage of whole optimization for complicated parts. Optimization of the blank initial contour line in hot stamping saves raw materials and cutting time and provides a vital reference for industry production.

Acknowledgments. This work was supported by the National Natural Science Foundation of China (Nos. 51205162 and 51275203). 
1. R. Kolleck, D. Steinhoefer, J. A. Feindt, and P. Bruneau, "Manufacturing methods for safety and structural body parts for lightweight body design," in: Proc. of the Conf. IDDRG (Sindelfingen, Germany, 2004), pp. 167-173.

2. M. Merklein and J. Lechler, "Investigation of the thermo-mechanical properties of hot stamping steels," J. Mater. Process. Technol., 177, No. 1, $452-455$ (2006).

3. Y. Q. Guo, J. L. Batoz, and H. Naceur, "Recent developments on the analysis and optimum design of sheet metal forming parts using a simplified inverse approach," Comput. Struct., 78, 133-148 (2000).

4. Y. Q. Guo, J. L. Batoz, J. M. Detraux, and P. Duroux, "Finite element procedures for strain estimations of sheet metal forming parts," Int. J. Numer. Meth. Eng., 30, No. 8, 1385-1401 (1990).

5. K. Son and H. Shim, "Optimal blank shape design using the initial velocity of boundary nodes," J. Mater. Process. Technol., 134, No. 1, 92-98 (2003).

6. X. Chen and R. Sowerby, "The development of ideal blank shapes by the method of plane stress characteristics," Int. J. Mech. Sci., 34, No. 2, 159-166 (1992).

7. M. H. Parsa, P. H. Matin, and M. M. Mashhadi, "Improvement of initial blank shape for intricate products using slip line method," J. Mater. Process. Technol., 145, No. 1, 21-26 (2004).

8. R. Sowerby, J. L. Duncan, and E. Chu, "The modeling of sheet metal stamping," Int. J. Mech. Sci., 28, No. 7, 415-430 (1986).

9. X. C. Lu and B. W. Liang, "Modeling of flange deformation of irregular drawn cups using a fluid analogy," Int. J. Mech. Sci., 28, No. 8, 491-497 (1986).

10. Z. T. Zhang and B. W. Liang, "Determination of blank shapes for drawing irregular cups using an electrical analogue method," Int. J. Mech. Sci., 28, No. 8, 499-503 (1986).

11. B. Oberpriller, L. Burkhardt, and B. Griesbach, "Benchmark 3 - Continuous Press Hardening," in: Proc. of the Conf. NUMISHEET'2008 (Interlaken, Switzerland, 2008), pp. 115-124. 